\title{
Faster and Energy-Efficient Signed Multipliers
}

\author{
B. Ramkumar and Harish M. Kittur \\ VLSI Division, School of Electronics Engineering, VIT University, Vellore 632014, Tamilnadu, India
}

Correspondence should be addressed to B. Ramkumar; bramvlsi@gmail.com

Received 13 December 2012; Accepted 22 April 2013

Academic Editor: Juan Sanchez-Garcia

Copyright (C) 2013 B. Ramkumar and H. M. Kittur. This is an open access article distributed under the Creative Commons Attribution License, which permits unrestricted use, distribution, and reproduction in any medium, provided the original work is properly cited.

\begin{abstract}
We demonstrate faster and energy-efficient column compression multiplication with very small area overheads by using a combination of two techniques: partition of the partial products into two parts for independent parallel column compression and acceleration of the final addition using new hybrid adder structures proposed here. Based on the proposed techniques, 8-b, 16-b, 32b, and 64-b Wallace (W), Dadda (D), and HPM (H) reduction tree based Baugh-Wooley multipliers are developed and compared with the regular W, D, H based Baugh-Wooley multipliers. The performances of the proposed multipliers are analyzed by evaluating the delay, area, and power, with $65 \mathrm{~nm}$ process technologies on interconnect and layout using industry standard design and layout tools. The result analysis shows that the 64-bit proposed multipliers are as much as $29 \%, 27 \%$, and $21 \%$ faster than the regular W, D, $\mathrm{H}$ based Baugh-Wooley multipliers, respectively, with a maximum of only $2.4 \%$ power overhead. Also, the power-delay products (energy consumption) of the proposed 16-b, 32-b, and 64-b multipliers are significantly lower than those of the regular BaughWooley multiplier. Applicability of the proposed techniques to the Booth-Encoded multipliers is also discussed.
\end{abstract}

\section{Introduction}

High-speed multiplication is a primary requirement of highperformance digital systems. In recent trends, the column compression multipliers are popular for high-speed computations due to their higher speeds $[1,2]$. The first column compression multiplier was introduced by Wallace in 1964 [3]. He reduced the partial product of $N$ rows by grouping into sets of three-row set and two-row set using $(3,2)$ counter and $(2,2)$ counter, respectively. In 1965, Dadda altered the approach of Wallace by starting with the exact placement of the $(3,2)$ counter and $(2,2)$ counter in the maximum critical path delay of the multiplier [4]. Three-dimensional minimization- (TDM-) based column compression approach was proposed in 1996 to perform fast multiplication [5]. Since the 2000s, a closer reconsideration of Wallace and Dadda multipliers has been done and proved that the Dadda multiplier is slightly faster than the Wallace multiplier and the hardware required for Dadda multiplier is lesser than the Wallace multiplier $[6,7]$. The HPM-based column compression was developed in 2006, and it has standard layout structure than Eriksson et al's multiplier [8]. The detailed case for HPM-based Baugh-Wooley multiplier against the BoothEncoded multipliers has been described in [9]. In this work, we implement the proposed techniques with the W, D, H based Baugh-Wooley multipliers, and the improved performance is compared with that of the same regular multipliers.

The Baugh-Wooley (BW) algorithm is a relatively straightforward way of doing signed multiplications [10]; Figure 1 illustrates the algorithm for an 8-bit case, where the partial-product bits have been reorganized as specified by Själander and Larsson-Edefors in his work [11]. The creation of the reorganized partial-product array comprises three steps: (i) the most significant bit (MSB) of the $N-1$ partialproduct rows and all bits of the last partial-product row, except its MSB, are inverted; (ii) a " 1 " is added to the Nth column; (iii) the MSB of the final result is inverted. The total delay of the multiplier can be split up into three parts: due to the partial-product generator (PPG), partial-product summation tree (PPST), and final CPA [12]. Of these, the dominant components of the multiplier delay are due to the PPST and the final adder. The relative delay due to the PPG is small. Therefore, a significant improvement in the speed of the multiplier can be achieved by reducing the delay in 


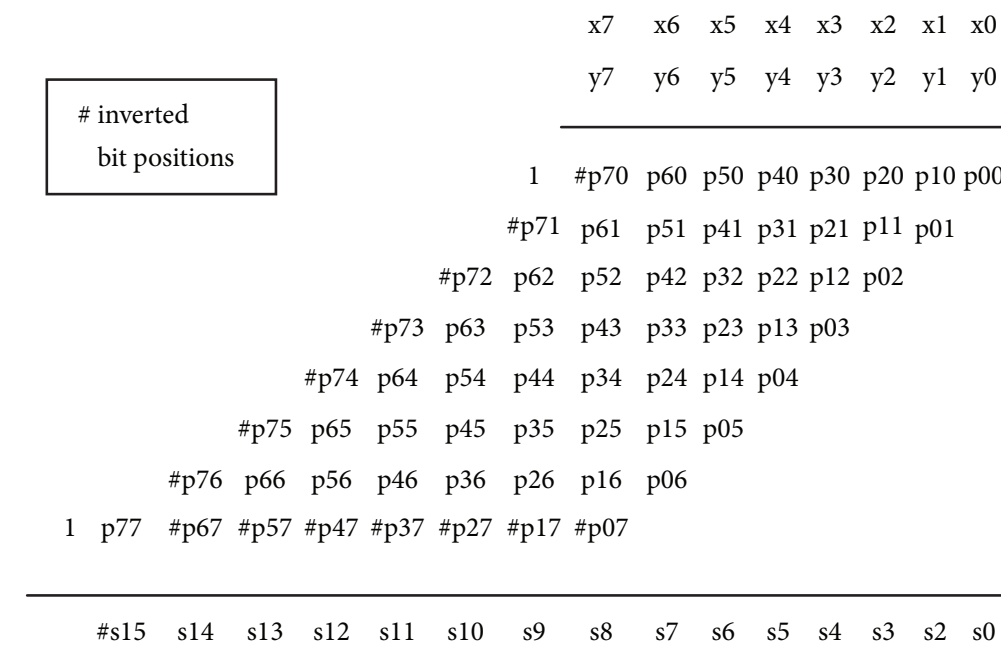

FIgURE 1: Illustration of an 8-bit Baugh-Wooley multiplication.

the PPST and the final adder stage of the multiplier. In this work, the delay of PPST is reduced by using two independent structures in the partial products. The proposed hybrid CPA, based on arrival profile aware design $[12,13]$ and the BEC (Binary to Excess-1 Converter) Logic [14, 15], computes the final products much faster. Arrival profile aware hybrid adders have been reported earlier $[12,13]$. Recently, further investigations on the same are reported in [16].

This paper is structured as follows. Sections 2 and 3 describe the design of parallel structures for the PPST and the design of hybrid final adder structure, respectively. Section 4 reports the ASIC implementation details and the simulation results. Finally, Section 5 summarizes the result analysis. Throughout the paper, it is assumed that the number of bits in the multiplier and multiplicand is equal.

\section{Design of Parallel Structures}

The multiplication process begins with the generation of all partial products in parallel using an array of AND gates. The next major steps in the design process are partitioning of the partial products and their reduction process. Each of these steps is elaborated in the following subsections.

2.1. Partitioning the Partial Products. We consider two $n$-bit (8-bit) operands of Baugh-Wooley multiplier partial products which form a matrix of $n$ rows and $2 n$ columns as shown in Figure 1. Initially for the partial product of Baugh-Wooley multiplication, we assign an integer as shown in Figure 2(a); for example, p00 is given an index 0, p10 an index 1, and so on. For convenience, we rearrange the partial products as shown in Figure 2(b). The two longest columns in the middle of the partial products contribute to the maximum delay in the PPST. Therefore, in this work, we split up the PPST into two parts as shown in the Figure 2(c), in which both parts share equal number of columns. That is, part0 consists of $n$ columns and partl also consists of $n$ columns. We then proceed to sum up each column of the two parts in parallel. The summation procedure adopted in this work is described in the next section.

2.2. The $W, D, H$ Based Reduction. Next, the partial products of each part are reduced to two rows by the using $(3,2)$ and $(2,2)$ counters based on the $\mathrm{W}, \mathrm{D}, \mathrm{H}$ reduction algorithm. The HPM-based reduction is shown in Figures 3 and 4. The grouping of 3-bits and 2-bits indicates $(3,2)$ and $(2,2)$ counters, respectively, and the different colors classify the difference between each column. The bit positions s0, 22, and 29 are added using $(3,2)$ counter to generate sum s2 and carry c2. The final two rows of each part are summed using a carry lookahead adder (CLA) to perform fast addition, and it forms the partial final products of a height of one-bit column, which is indicated at the bottom of Figures 3 and 4 .

The two parallel structures in Figures 3 and 4 based on the HPM method are shown in Figure 5, where HA, FA, p0, p1, and p denote half adder $((2,2)$ counter $))$, full adder $((3,2)$ counter $)$, partial final product from part0, partial final product from partl, and final product, respectively. The numerals residing on the HA and FA indicate the position of partial products. The outputs of part 0 and partl are computed independently in parallel, and those values are added using a high-speed hybrid final adder to get the final product.

However, before we proceed to carry out the final addition with the proposed hybrid adder, we first carry out the final addition with the faster adder of CLA for both the unpartitioned W, D, H Baugh-Wooley multiplier and the partitioned W, D, H Baugh-Wooley multiplier. This enables us to evaluate and analyze the effect of partitioning the PPST into two parts. The simulation results and their comparison are listed in Tables 1, 2, and 3, in these tables a negative percentage indicates overhead and a positive percentage indicates a reduction/improvement with reference to the compared multiplier. The comparison shows the percentage improvement 
TABLE 1: Partitioned Wallace performance.

\begin{tabular}{lccccccrrr}
\hline & \multicolumn{3}{c}{ Regular Wallace multiplier with CLA } & \multicolumn{2}{c}{ Partitioned Wallace multiplier with CLA } & \multicolumn{3}{c}{ Performance comparison (\%) } \\
Word size & Delay $(\mathrm{ns})$ & Area $\left(\mu \mathrm{m}^{2}\right)$ & Power $(\mu \mathrm{W})$ & Delay $(\mathrm{ns})$ & Area $\left(\mu \mathrm{m}^{2}\right)$ & Power $(\mu \mathrm{W})$ & Delay & Area & Power \\
\hline 16 & 1.84 & 3,983 & 0.66 & 1.76 & 4,093 & 0.69 & 4.34 & -2.76 & -4.54 \\
32 & 2.52 & 14,398 & 3.21 & 2.30 & 14,665 & 3.33 & 8.73 & -1.85 & -3.73 \\
64 & 3.29 & 53,896 & 16.86 & 2.92 & 54,449 & 17.34 & 11.24 & -1.02 & -2.84 \\
\hline
\end{tabular}

TABle 2: Partitioned Dadda performance.

\begin{tabular}{|c|c|c|c|c|c|c|c|c|c|}
\hline \multicolumn{4}{|c|}{ Regular Dadda multiplier with CLA } & \multicolumn{3}{|c|}{ Partitioned Dadda multiplier with CLA } & \multicolumn{3}{|c|}{ Performance comparison (\%) } \\
\hline Word size & Delay (ns) & Area $\left(\mu \mathrm{m}^{2}\right)$ & Power $(\mu \mathrm{W})$ & Delay (ns) & Area $\left(\mu \mathrm{m}^{2}\right)$ & Power $(\mu \mathrm{W})$ & Delay & Area & Power \\
\hline 16 & 1.69 & 3,843 & 0.61 & 1.66 & 3,986 & 0.65 & 1.77 & -3.72 & -6.55 \\
\hline 32 & 2.41 & 13,804 & 3.14 & 2.23 & 14,083 & 3.26 & 7.46 & -2.02 & -3.82 \\
\hline 64 & 3.11 & 51,885 & 16.12 & 2.86 & 50,517 & 16.58 & 8.03 & 2.63 & -2.85 \\
\hline
\end{tabular}

$$
\begin{aligned}
& \begin{array}{lllllllll}
8 & 7 & 6 & 5 & 4 & 3 & 2 & 1 & 0
\end{array} \\
& \begin{array}{llllllll}
16 & 15 & 14 & 13 & 12 & 11 & 10 & 9
\end{array} \\
& \begin{array}{llllllll}
24 & 23 & 22 & 21 & 20 & 19 & 18 & 17
\end{array} \\
& \begin{array}{llllllll}
32 & 31 & 30 & 29 & 28 & 27 & 26 & 25
\end{array} \\
& \begin{array}{llllllll}
40 & 39 & 38 & 37 & 36 & 35 & 34 & 33
\end{array} \\
& \begin{array}{llllllll}
48 & 47 & 46 & 45 & 44 & 43 & 42 & 41
\end{array} \\
& \begin{array}{llllllll}
56 & 55 & 54 & 53 & 52 & 51 & 50 & 49
\end{array} \\
& \begin{array}{lllllllll}
65 & 64 & 63 & 62 & 61 & 60 & 59 & 58 & 57
\end{array} \\
& \begin{array}{llllllllllllllll}
65 & 64 & 56 & 48 & 40 & 32 & 24 & 8 & 7 & 6 & 5 & 4 & 3 & 2 & 1 & 0
\end{array} \\
& \begin{array}{lllllllllllll}
63 & 55 & 47 & 39 & 31 & 16 & 15 & 14 & 13 & 12 & 11 & 10 & 9
\end{array} \\
& \begin{array}{lllllllllll}
62 & 54 & 46 & 38 & 23 & 22 & 21 & 20 & 19 & 18 & 17
\end{array} \\
& \begin{array}{lllllllll}
61 & 53 & 45 & 30 & 29 & 28 & 27 & 26 & 25
\end{array} \\
& \begin{array}{lllllll}
60 & 52 & 37 & 36 & 35 & 34 & 33
\end{array} \\
& \begin{array}{lllll}
59 & 44 & 43 & 42 & 41
\end{array} \\
& \begin{array}{lll}
51 & 50 & 49
\end{array} \\
& 58 \quad 57
\end{aligned}
$$

\begin{tabular}{|c|c|c|c|c|c|c|c|c|c|c|c|c|c|c|}
\hline \multirow[t]{8}{*}{$\begin{array}{ll}65 & 64\end{array}$} & 56 & 48 & 40 & 32 & 24 & 8 & 7 & 6 & 5 & 4 & 3 & 2 & 1 & 0 \\
\hline & 63 & 55 & 47 & 39 & 31 & 16 & 15 & 14 & 13 & 12 & 11 & 10 & 9 & \\
\hline & & 62 & 54 & 46 & 38 & 23 & 22 & 21 & 20 & 19 & 18 & 17 & & \\
\hline & & & 61 & 53 & 45 & 30 & 29 & 28 & 27 & 26 & 25 & & & \\
\hline & & & & 60 & 52 & 37 & 36 & 35 & 34 & 33 & & & & \\
\hline & & & & & 59 & 44 & 43 & 42 & 41 & & & & & \\
\hline & Part & & & & & 51 & 50 & 49 & & & & Part2 & & \\
\hline & & & & & & 58 & 57 & & & & & & & \\
\hline
\end{tabular}

(b)

(c)

FIGURE 2: Partitioning the partial products: (a) partial-product array diagram for $8 * 8$ multiplier, (b) an alternative representation, and (c) partitioned structure of multiplier showing part0 and part1. 
TABle 3: Partitioned HPM performance.

\begin{tabular}{lccccccrrr}
\hline & \multicolumn{3}{c}{ Regular HPM multiplier with CLA } & \multicolumn{2}{c}{ Partitioned HPM multiplier with CLA } & \multicolumn{3}{c}{ Performance comparison (\%) } \\
Word size & Delay $(\mathrm{ns})$ & Area $\left(\mu \mathrm{m}^{2}\right)$ & Power $(\mu \mathrm{W})$ & Delay $(\mathrm{ns})$ & Area $\left(\mu \mathrm{m}^{2}\right)$ & Power $(\mu \mathrm{W})$ & Delay & Area & Power \\
\hline 16 & 1.75 & 3,848 & 0.63 & 1.72 & 4,025 & 0.67 & 1.71 & -4.59 & -6.34 \\
32 & 2.48 & 13,817 & 3.16 & 2.36 & 14,173 & 3.33 & 4.80 & -2.57 & -5.37 \\
64 & 3.18 & 51,912 & 16.35 & 2.99 & 50,673 & 16.72 & 5.97 & 2.38 & -2.26 \\
\hline
\end{tabular}

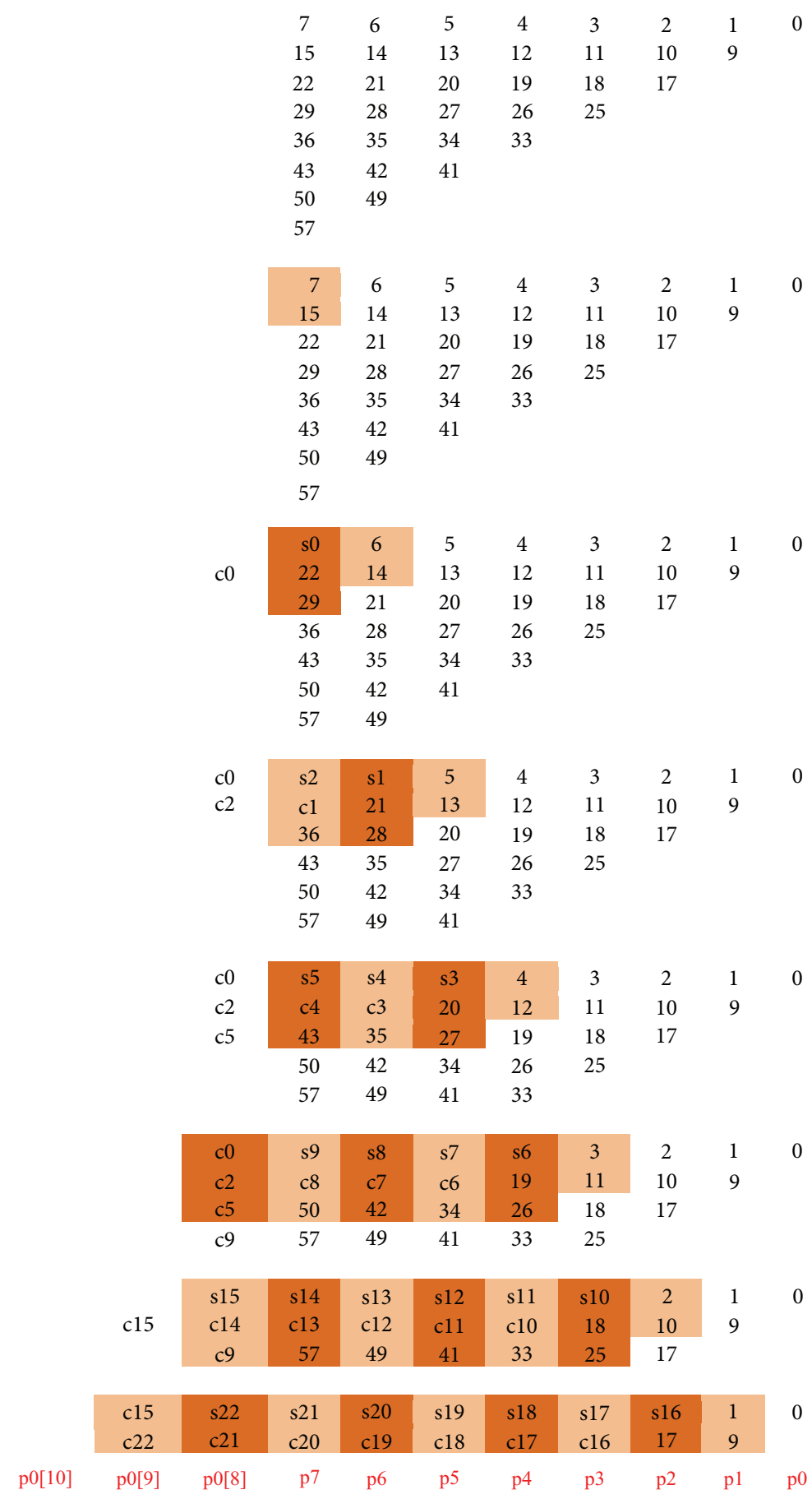

FIGURE 3: Reduction of the partial products of part0 based on the HPM reduction approach. 


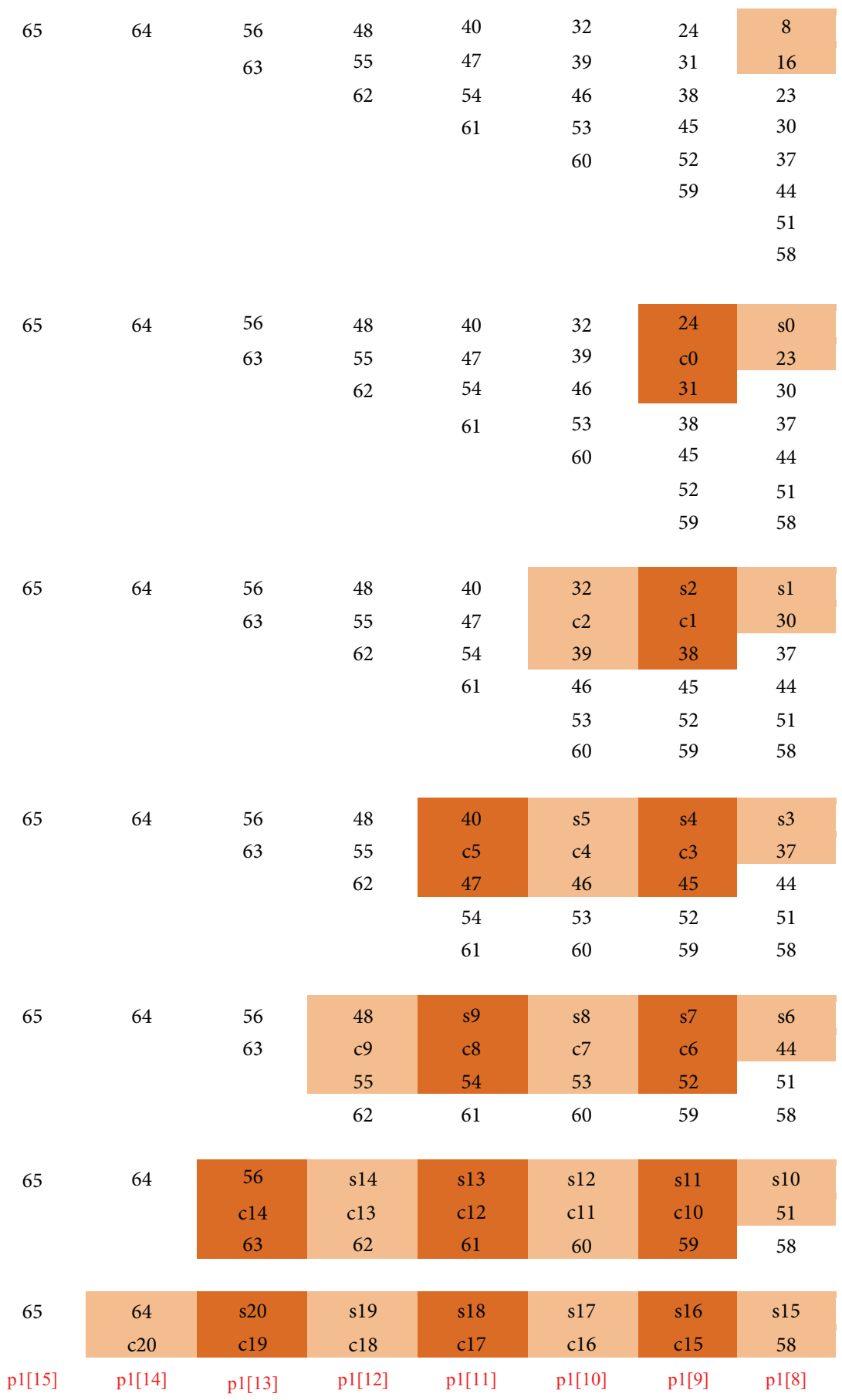

FIGURE 4: Reduction of the partial products of partl based on the HPM reduction approach.

and overhead in delay, area, and power of the partitioned multipliers with respect to the unpartitioned multiplier.

It can be seen that there is $4.3 \%$ improvement in the speed for $16-\mathrm{b}$ and $11.2 \%$ for $64-\mathrm{b}$ size. The speed limitation in lower bit size multipliers is due to the greater difference between input arrival profile to the final CPA from part0 and part1. But with the increase in the word size, this difference becomes lesser and the improvement in the speed of the partitioned multipliers increases. There is maximum of $11 \%, 8 \%$, and $6 \%$ speed improvement for 64-b W, D, H Baugh-Wooley multipliers with $1 \%$ area overhead. Having clearly demonstrated the reduction in the delay of the multipliers due to the partitioning of the partial products, we now proceed to further enhance the speed of the proposed multiplier. There is maximum of $6 \%$ to $7 \%$ power overhead in W, D, $\mathrm{H}$ based BaughWooley multiplier, and this is due to the use of CLA as CPA in each part. But this power overhead is interestingly reduced by proposed hybrid CPA which is elaborated in the next section. 


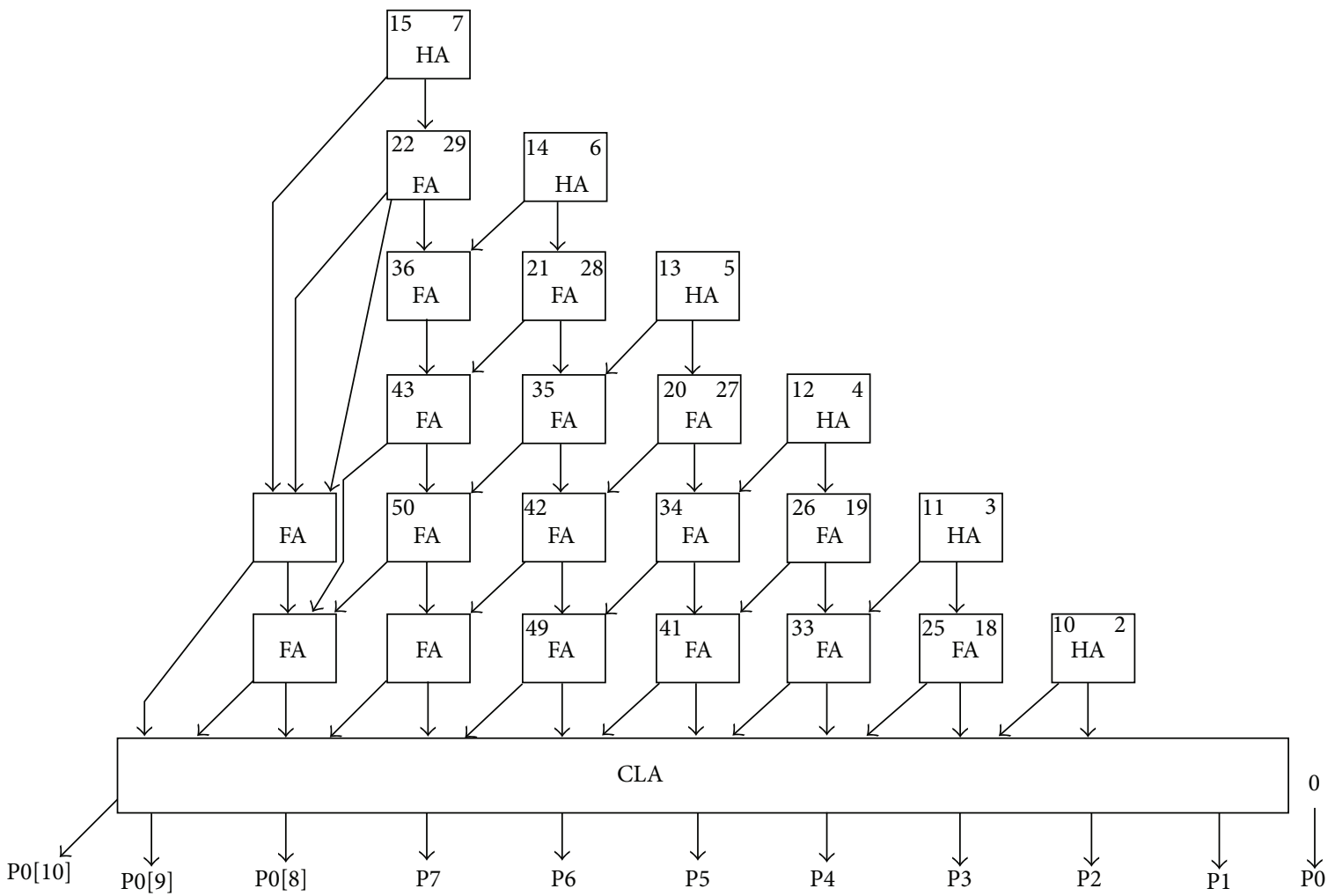

(a)

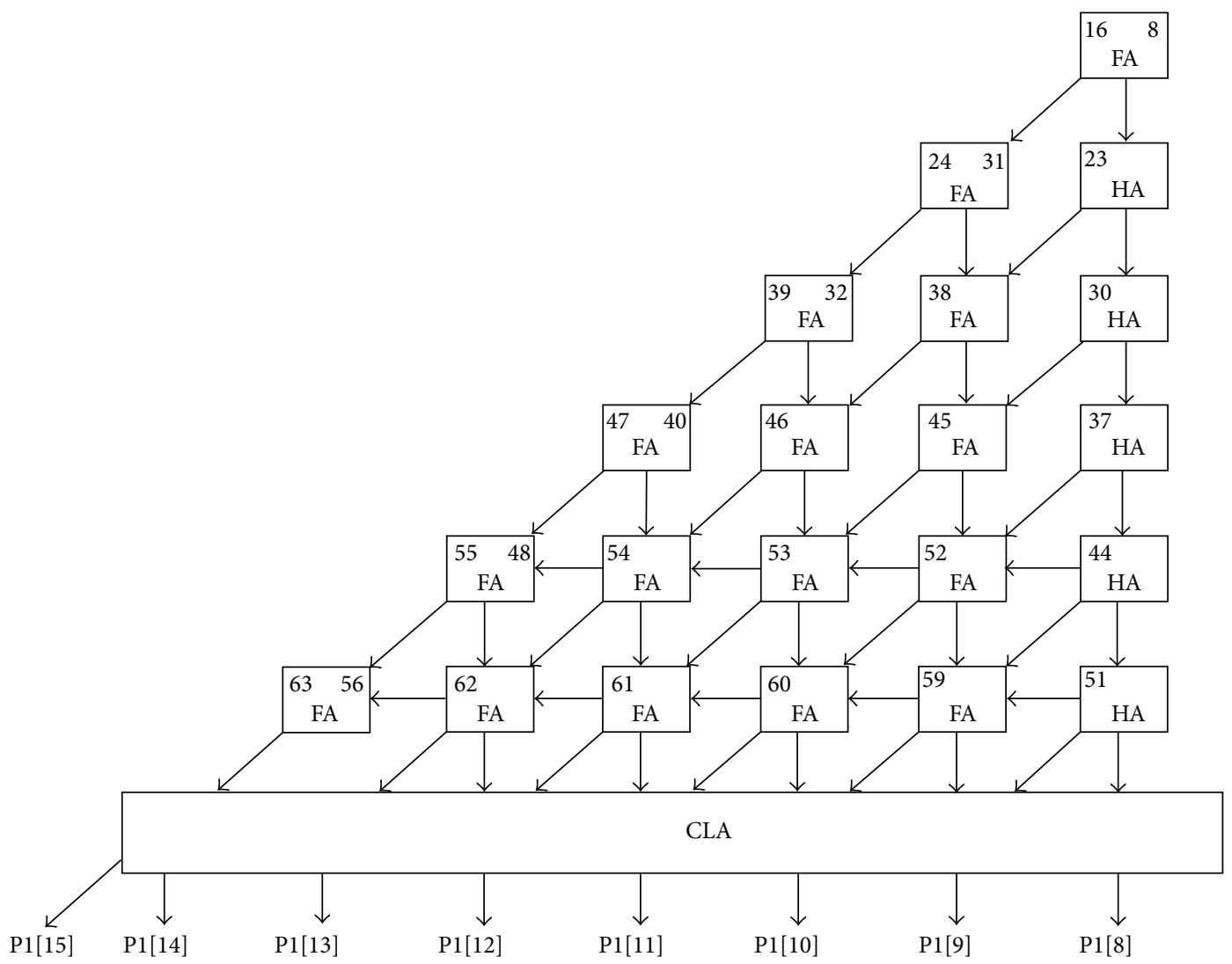

(b)

FIgURE 5: The HPM-based implementation: (a) implementation of part0; (b) implementation of part1. 


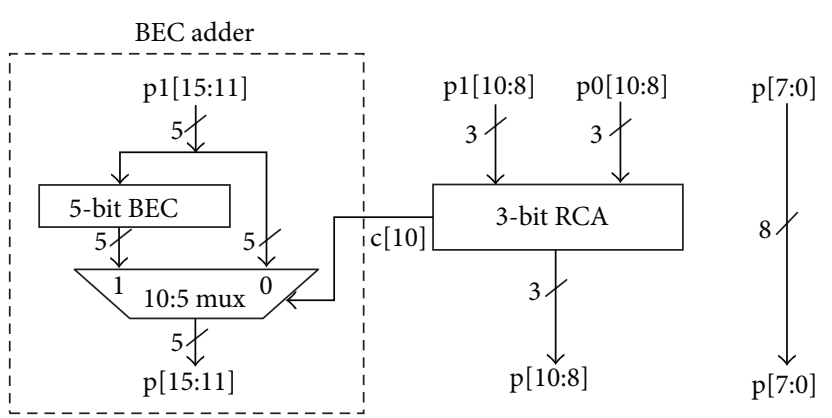

Figure 6: Hybrid final adder of 8-b multiplier.

\section{The Hybrid Final Adder Design}

In previous works, the hybrid final adder designs used to achieve the faster performance in parallel multipliers were made up of CLA (carry lookahead adder) and CSLA (carry select adder) $[12,13]$. But due to the structure of the CSLA, it occupies more chip area and power than other adders. Thus to achieve the optimal performance, the proposed hybrid adder in this work uses BEC logic for fast summation of uneven input arrival time of the signals originating from the PPST. The BEC adder provides faster performance than carry save adder (CSA) and it consumes less area, low power than the carry select adder (CSLA) [14, 15].

3.1. Hybrid Adder for 8-b Multiplier. Once each part of the partial products has been reduced to height of one bit column, we get the final partial products as follows:

$$
\mathrm{p} 0[10] \mathrm{p} 0[9] \mathrm{p} 0[8]
$$

$$
\begin{aligned}
& \text { p7 p6 p5 p4 p3 p2 p1 p0 } \\
& \text { p1[15] p1[14] p1[13] P1[12] p1[11] p1[10] p1[9] p1[8]. }
\end{aligned}
$$

The p0[10:8] are the exceeding carry bits of part0 and $\mathrm{p} 1[15]$ is the carry bit of partl. The $\mathrm{p}[7: 0]$ of part0 are directly assigned as the final products. To find the remaining $\mathrm{p}[15: 8]$, we use the RCA and the BEC as shown in Figure 6.

The $\mathrm{p} 0[10: 8]$ and $\mathrm{p} 1[10: 8]$ are added using 3-bit RCA which finds $\mathrm{p}[10: 8]$. To obtain the remaining $\mathrm{p}[15: 11]$, the p1 [15:11] are assigned to the input of 5-bit BEC, which produce the two partial results p1[15:11] with Cin of " 0 " and the 5bit BEC output with the Cin of "1." Depending on the Cout of RCA (c[10]), the mux provides the final $\mathrm{p}$ [15:11] without having to ripple the carry through $\mathrm{p} 1[15: 11]$.

The 8-bit multiplier uses a 5-bit BEC in the final adder, but for the large bit sized multipliers requires multiple BEC, and each of them requires the selection input from the carry output of the preceding BEC. Therefore, to generate the carry output from the BEC, an additional block is developed which is called BECWC. The detailed structures of the 5-bit BEC without carry (BEC) and with carry (BECWC) are shown in Figures 7 (a) and 7(b). The BEC gets $n$ inputs and generates $n$ output; the BECWC gets $n$ input and generates $n+1$ output to give the carry output as the selection input of the next stage mux used in the final adder design of 16-b, 32-b,
TABLE 4: Function table of 5-BIT BEC and BECWC.

\begin{tabular}{cccc}
\hline $\begin{array}{c}\text { Input } \\
\mathrm{b}[4: 0]\end{array}$ & BEC without carry & \multicolumn{2}{c}{ BEC with carry } \\
\hline 00000 & $\mathrm{x}[4: 0]$ & $\mathrm{cy}$ & $\mathrm{x}[4: 0]$ \\
$\vdots$ & 00001 & 0 & 00001 \\
11110 & $\vdots$ & $\vdots$ & $\vdots$ \\
11111 & 11111 & 0 & 11111 \\
\hline
\end{tabular}

and 64-b multipliers. The function table of BEC and BECWC is shown in Table 4.

3.2. Variable-Size Hybrid Adder. The variable size of adder blocks always leads to faster performance than a fixed-size block adder [2, 17]; we, therefore, break down the ripple of gates in the BEC into variable-size groups according to the $\log _{2} n$ method. Based on this approach, the final adder designs for 16-b, 32-b, and 64-b multipliers are shown in Figure 8.

In BECWC, the mux is getting $n$-bits of data input as it is input for selection input " 0 " side and $n+1$-bits of data input from the BECWC output for selection input " 1 " side. Thus to make equal size of the inputs to the mux, the one-bit " 0 " is appending with the $n$-bits of the data input as "MSB" (most significant bit).

To analyze independently the effect of the proposed hybrid adder, the partitioned multiplier with CLA final adder is compared with the partitioned multiplier along with the proposed hybrid adder. The simulation results of partitioned W, D, H Baugh-Wooley multipliers with hybrid CPA are listed as first column in Tables 5, 6, and 7. The performance of hybrid CPA (comparison between the partioned multipliers with CLA and partitioned multipliers with hybrid CPA) and overall performance of proposed techniques (comparison between unpartitioned multiplier with CLA and partitioned multiplier with hybrid CPA) are listed as second column and third column, respectively, in Tables 5 to 7 . The result analysis clearly shows that the speed increases with the word size of the multiplier. The hybrid CPA improves the speed of the W, D, H Baugh-Wooley multipliers by $19 \%, 20 \%, 15 \%$, respectively, for 64-b size without area and power overhead. The overall improved performance is elaborated in result summary.

\section{ASIC Implementation and Simulation Results}

The ASIC implementation of the proposed design follows the cadence design flow. The design has been developed using Verilog-HDL and synthesized in Encounter RTL compiler using typical libraries of TSMC $65 \mathrm{~nm}$ technology. The Cadence SoC Encounter is adopted for Placement \& Routing (P\&R) (Encounter User Guide 2008). Parasitic extraction is performed using Encounter Native RC extraction tool. The extracted parasitic RC (SPEF format) is back annotated to Common Timing Engine in Encounter Platform for static timing analysis. 
TABLE 5: Improved performance by hybrid CPA and overall performance in Wallace multiplier.

\begin{tabular}{lcccccccrr}
\hline \multicolumn{2}{c}{ Partitioned Wallace multiplier with hybrid CPA } & \multicolumn{3}{c}{ Performance of hybrid CPA } & \multicolumn{3}{c}{ Overall performance } \\
Word size & Delay $(\mathrm{ns})$ & Area $\left(\mu \mathrm{m}^{2}\right)$ & Power $(\mu \mathrm{W})$ & Delay & Area & Power & Delay & Area & Power \\
\hline 16 & 1.58 & 4,137 & 0.71 & 10.22 & -1.07 & -2.89 & 14.13 & -3.86 & -7.57 \\
32 & 1.98 & 14,758 & 3.39 & 13.91 & -0.63 & -1.80 & 21.42 & -2.50 & -5.60 \\
64 & 2.35 & 54,225 & 17.28 & 19.52 & 0.41 & 0.34 & 28.57 & -0.61 & -2.49 \\
\hline
\end{tabular}

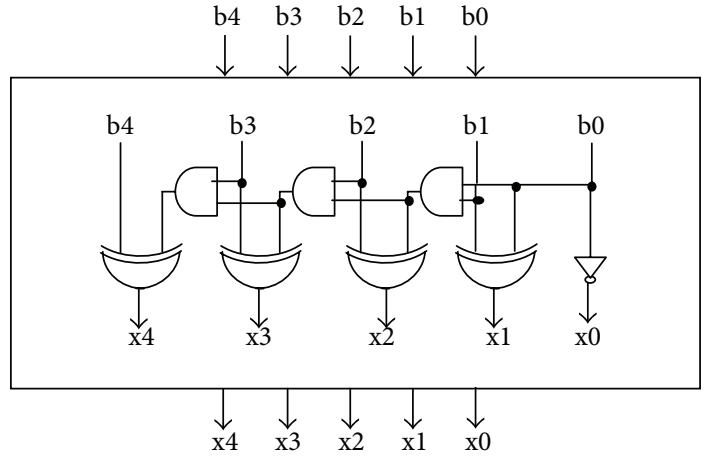

(a)

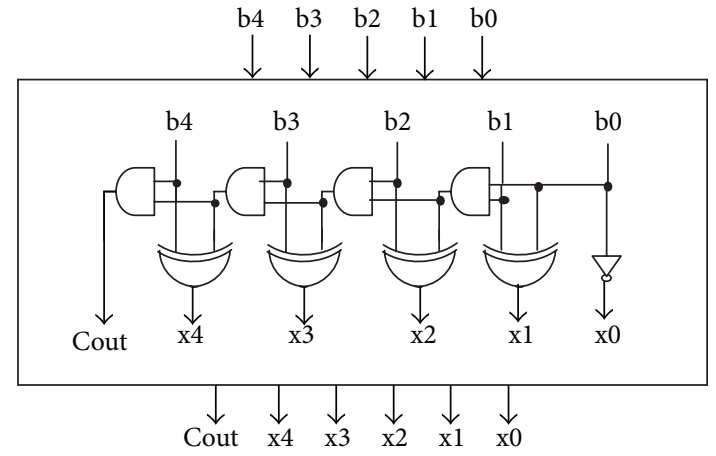

(b)

FIgURE 7: The 5-bit Binary to Excess-1 Code Converter: (a) BEC (without carry); (b) BECWC (with carry).
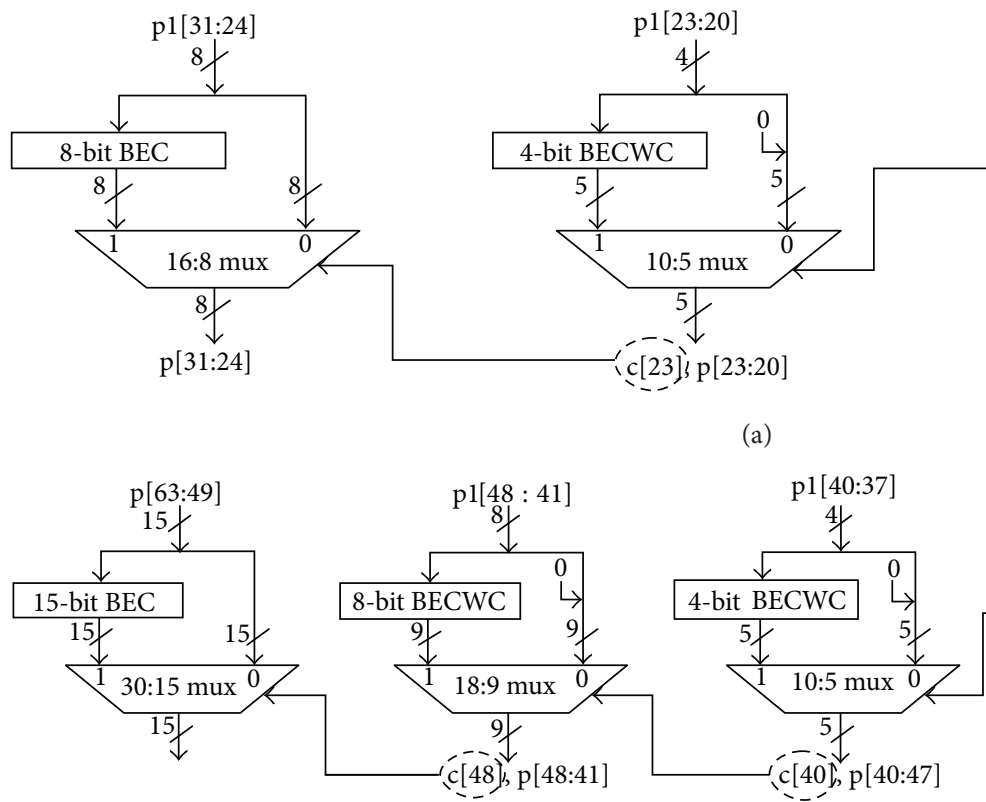

(b)
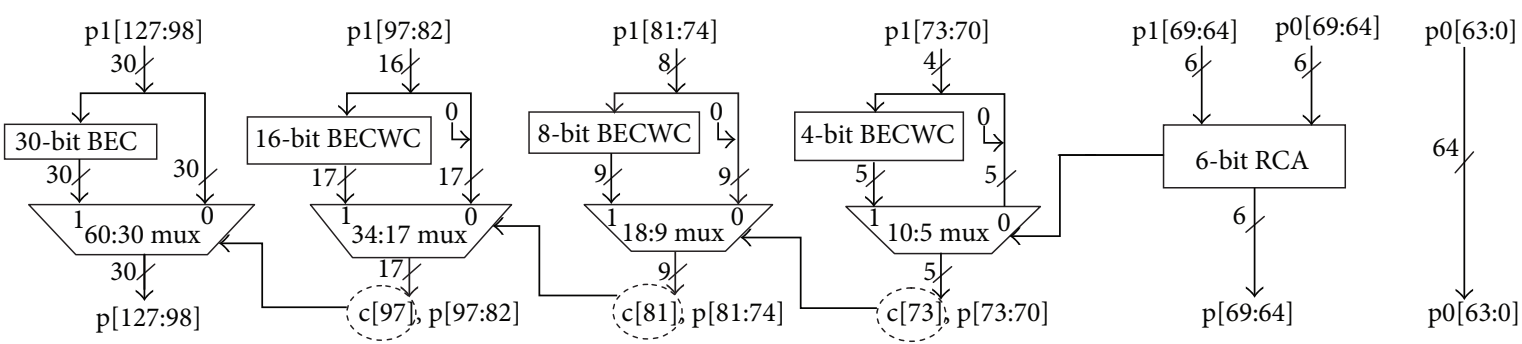

(c)

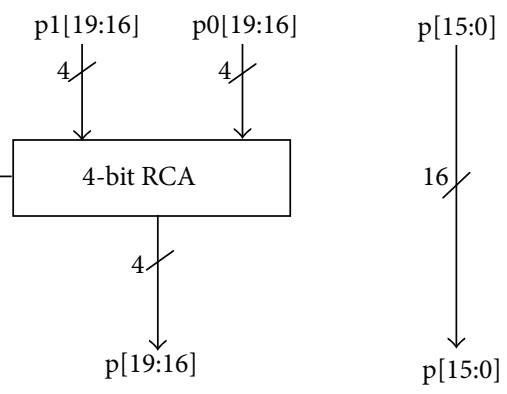

Figure 8: Hybrid final adder: (a) for 16-b multiplier, (b) for 32-b multiplier, and (c) for 64-b multiplier. 
TABLE 6: Improved performance by hybrid CPA and overall performance in Dadda multiplier.

\begin{tabular}{lccccccccc}
\hline & \multicolumn{2}{c}{ Partitioned Dadda multiplier with hybrid CPA } & \multicolumn{3}{c}{ Performance of hybrid CPA } & \multicolumn{3}{c}{ Overall performance } \\
Word size & Delay $(\mathrm{ns})$ & Area $\left(\mu \mathrm{m}^{2}\right)$ & Power $(\mu \mathrm{W})$ & Delay & Area & Power & Delay & Area & Power \\
\hline 16 & 1.49 & 4,028 & 0.66 & 10.24 & -1.05 & -1.53 & 11.8 & -4.81 & -8.19 \\
32 & 1.92 & 14,100 & 3.29 & 13.90 & -0.12 & -0.92 & 20.3 & -2.14 & -4.77 \\
64 & 2.27 & 49,877 & 16.47 & 20.62 & 1.26 & 0.66 & 27.0 & 3.87 & -2.17 \\
\hline
\end{tabular}

TABLE 7: Improved performance by hybrid CPA and overall performance in HPM multiplier.

\begin{tabular}{lcccccccrr}
\hline & \multicolumn{2}{c}{ Partitioned HPM multiplier with hybrid CPA } & \multicolumn{3}{c}{ Performance of hybrid CPA } & \multicolumn{3}{c}{ Overall performance } \\
Word size & Delay $(\mathrm{ns})$ & Area $\left(\mu \mathrm{m}^{2}\right)$ & Power $(\mu \mathrm{W})$ & Delay & Area & Power & Delay & Area & Power \\
\hline 16 & 1.59 & 4,071 & 0.70 & 7.55 & -1.14 & -4.47 & 9.14 & -5.79 & -11.11 \\
32 & 2.10 & 14,266 & 3.38 & 11.01 & -0.65 & -1.50 & 15.32 & -3.24 & -6.96 \\
64 & 2.52 & 50,301 & 16.65 & 15.71 & 0.73 & 0.41 & 20.75 & 3.10 & -1.83 \\
\hline
\end{tabular}

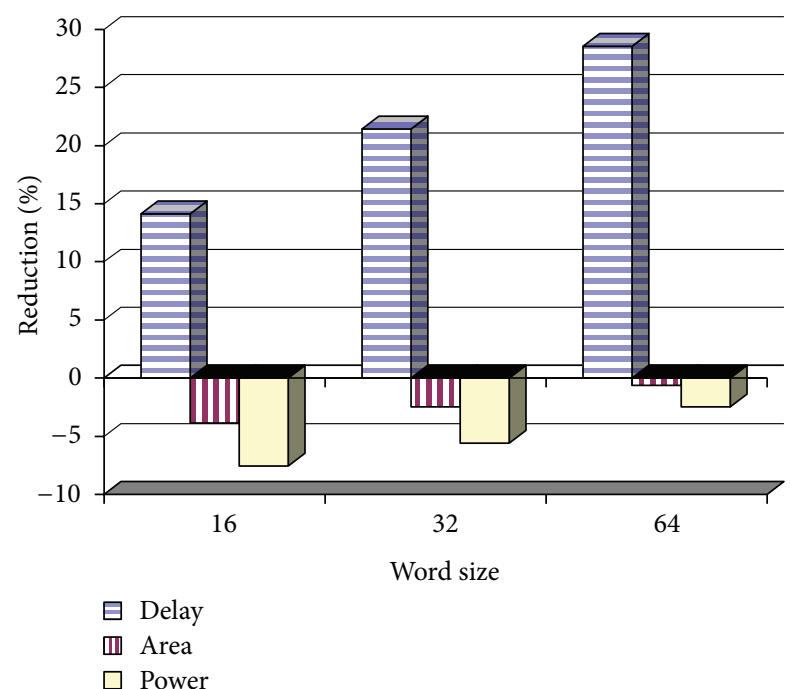

(a)

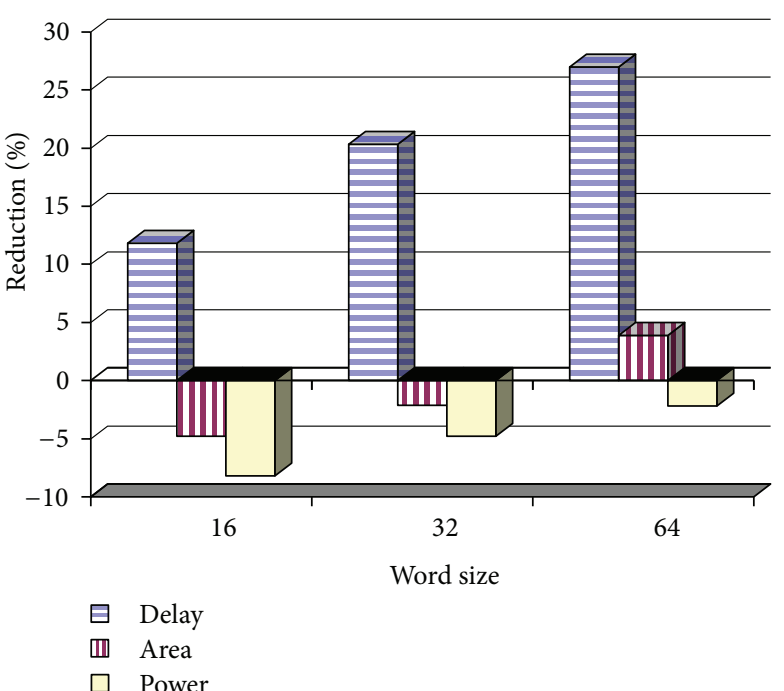

(b)

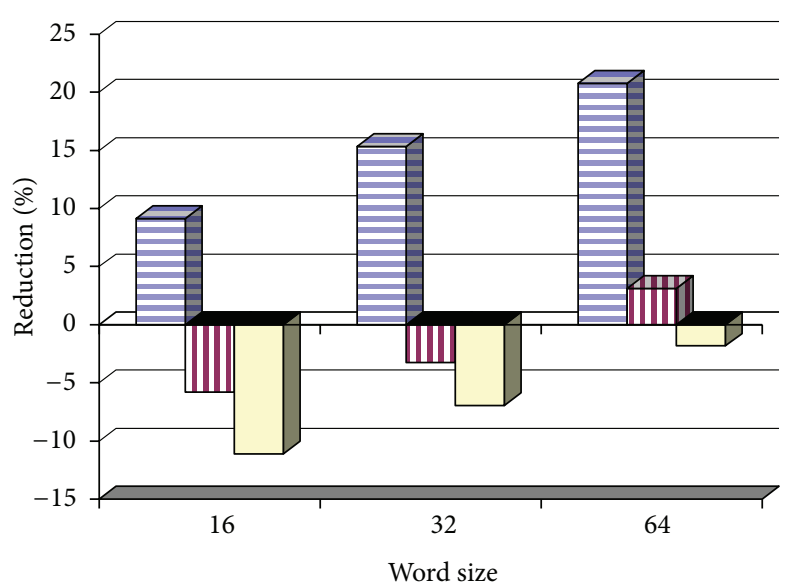

$\boxminus$ Delay
$\square$ Area
$\square$ Power

FIGURE 9: Overall performance of the proposed multipliers: (a) Wallace, (b) Dadda, and (c) HPM. 
In order to approximate typical signal arrival times and drive strengths, D flip-flops are used on the primary inputs. These flip-flops drive multiple buffers to distribute input signals to $N^{2}$ AND gates, where $N$ is the multiplier word size. Delay simulations were performed for each cell library to resolve the maximum number of buffers that a single D flip-flop can drive and the maximum number of AND gate inputs that a single buffer can drive. The Common Timing Engine used for timing simulation which takes as inputs a design's netlist, cell library process information, parasitic resistance and capacitance data, and simulation environment parameters such as temperature and voltage. All of the timing analysis is performed at the nominal voltage level $0.9 \mathrm{~V}$, for the $65 \mathrm{~nm}$ process technology. Temperature was set at $25^{\circ} \mathrm{C}$. The worst case delays of the multipliers are examined with back-annotation of parasitic resistances and capacitances extracted from the layouts. Each standard cell library used for this design includes LEF (Library Exchange Format) files and timing files. A LEF file contains the physical information for a process technology as well as geometric abstracts of all of the cells. All of the timing files used for this research is for the nominal temperature, voltage, and process corner, often named "typical.lib."

The power simulations were performed using Virtuoso UltraSim which takes as inputs a design's netlist, RC parasitics file in SPEF format, process technology information, temperature and voltage, and a vector stimulus file. For each word size of the multiplier, the VCD (value changed dump) data is generated for all possible input conditions and imported the same to power simulation tool. All the power simulations were performed at the nominal voltage level of $0.9 \mathrm{~V}$ for the $65 \mathrm{~nm}$ process technology. The simulation temperature was set at $25^{\circ} \mathrm{C}$. Area estimate is based on total cell area of the design. All the multipliers were placed and routed using NanoPlace and NanoRoute of Cadence's Encounter platform. Though five or more layers of metal were available for each process, the 8 by 8 multipliers were routed using three layers of metal and the large 64 by 64 multipliers were routed using four layers of metal. In this work, we have used the same technology and similar design flow for all the designs including the conventional designs used for comparison of the delay, area, and power characteristics.

\section{Result Summary}

The comparison between the unpartitioned multipliers with CLA and partitioned multipliers with hybrid CPA is listed as third column in Tables 5 to 7 . These overall performances are plotted in Figure 9. It summarizes the enhanced performance of the proposed techniques and exhibits that the area of the partitioned multipliers with hybrid CPA is maximum of $5.7 \%$ higher than the unpartitioned multipliers with CLA in $16-b$ word size. But with increasing word size, the area overhead reduces. It is clear that the area overhead of the proposed techniques continuously decreases with increasing word size and is only $0.6 \%$ overhead and $3.9 \%, 3.1 \%$ improvement for the 64-b W, D, H Baugh-Wooley multipliers, respectively.
The power consumption of the proposed multiplier is $11 \%$ more than regular multipliers for the 16 -b word size. With increasing word size, the power requirement for the proposed techniques is reduced. Thus the 64-b partitioned W, D, H Baugh-Wooley multipliers with hybrid CPA requires only $2.5 \%, 2.2 \%$, and $1.9 \%$, respectively. The percentage overhead of the power-delay products (PDPs) of the proposed multipliers with respect to the regular multipliers is plotted in Figure 10. Negative values indicate an overhead and positive values a reduction. The PDP values increase with the word size and achieved maximum of $27 \%, 25 \%$, and $19 \%$ reduction in the PDP for the 64-b W, D, H, respectively. The delay values clearly indicate that the proposed techniques much improve the speed of multiplication, also with increasing word size the percentage reduction of the delay increases. Thus, the speed is significantly improved by $29 \%, 27 \%$, and $21 \%$ for the $64-b$ W, D, H multipliers, respectively.

Though the main goal of this work is to demonstrate the faster and energy-efficient column compression multiplication and not make a comparison of the Wallace, Dadda, and HPM based multipliers, a comparison of the proposed three multipliers shows that for all bit sizes, the Dadda based multipliers are the fastest, consume least power, and therefore also have the lowest PDP. The HPM is based on the Dadda reduction scheme but a direct comparison of the original Dadda with the HPM has not been reported in [8]. A comparison in terms of the TOPS/W (Tera Operations per Watt) in the $65 \mathrm{~nm}$ shows that the proposed Dadda based multipliers have the highest TOPS/W for all the bit sizes where for 16-b: 1017 TOPS/W, 32-b: 158 TOPS/W, and 64-b: 27 TOPS/W. Implementation of the proposed multipliers in the $32 \mathrm{~nm}$ or $22 \mathrm{~nm}$ nodes could lead to much higher values of TOPS/W.

\section{Modified-Booth Multiplier Evaluation}

Booth's algorithm is another signed multiplication algorithm that multiplies two signed binary numbers. Here, the partial products of the multipliers are generated by using Modified Booth Encoding (MBE) algorithm which reduce the number of partial product rows to $n / 2+1$, thus reducing the size and enhancing the speed of the reduction tree [18]. Later, some approaches have been proposed to generate more regular partial product arrays with $n / 2$ rows for the MBE multipliers; thus the area, delay, and power consumption of the reduction tree, as well as the whole MBE multiplier, have been reduced [19].

In this work, in order to explore the applicability of the proposed techniques to the MBE multipliers, we have implemented the MBE with the recent HPM-based reduction. The experimental results for the 32-b HPM-based MBE multiplier, without and with the techniques proposed in this work are shown in Table 8 . It shows $11 \%$ speed improvement than the regular MBE multiplier with $10 \%$ power overhead. Referring to the results depicted in Figures 9 and 10 with increasing bit size, the speed improvement will increase, power overhead decrease, and the PDP reduce. The MBE in Table 8 has a rating of about 82 TOPS/W $\pm \% 1$ which is 
TABLE 8: Performance of 32-b MBE multiplier.

\begin{tabular}{lcccc}
\hline Word size & Multiplier & Delay $(\mathrm{ns})$ & Area $\left(\mu \mathrm{m}^{2}\right)$ & Power $(\mu \mathrm{W})$ \\
\hline \multirow{2}{*}{32} & Regular & 2.39 & 14,966 & 5.06 \\
& Proposed & 2.19 & 15,721 & 5.58 \\
\hline
\end{tabular}

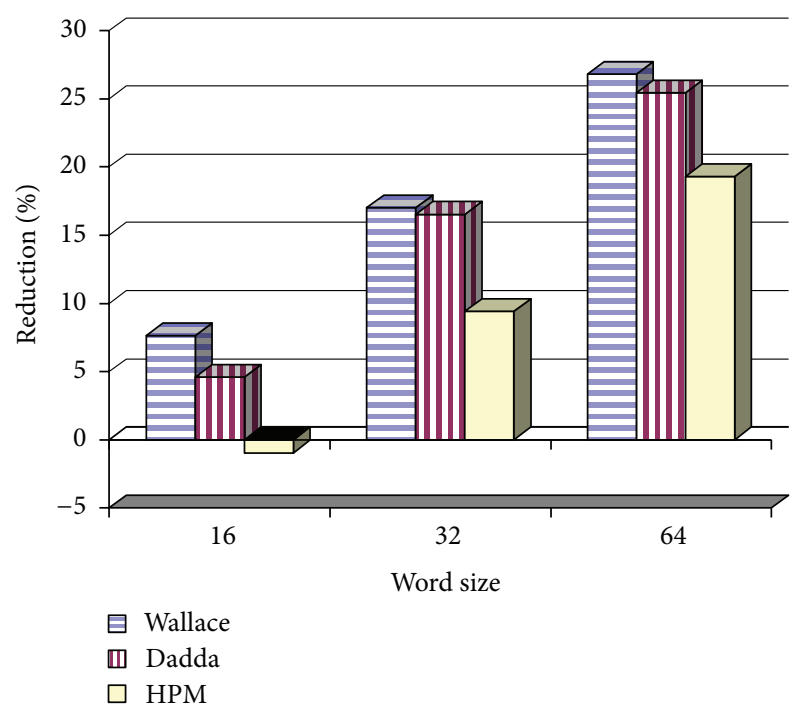

FIGURE 10: Power-delay-product comparison.

nearly half of the proposed 32-b Dadda based Baugh-Wooly multiplier.

\section{Conclusion}

We have successfully achieved faster column compression and fast final addition using hybrid final adder structure. With increasing word size, the percentage reduction of the delay increases; at the same time the percentage overhead of the area and power decreases. Actually, there is area reduction in case of the proposed 64-b D, $\mathrm{H}$ multipliers. The proposed 16-b, 32-b, and 64-b multipliers have PDP lower than the original multipliers and are, therefore, energy efficient. We have good reasons to believe that for bit sizes greater than or equal to 128 , significant speeds can be achieved without any area or power overhead; that is, the 128-bit multiplier would be not only fast but also area, power, and energy efficient. The speed improvements are significant. Also, we have proved that the proposed techniques improve the performance of different column compression multipliers. These design techniques can be implemented with any type of parallel multipliers and even the MBE multipliers of bit sizes greater than 32-b to achieve faster performance without significant area and power overhead.

\section{Disclosure}

This work was carried out at the Integrated Circuit Design Laboratories, VIT University, Vellore, India.

\section{References}

[1] K. C. Bickerstaff, Optimization of Column Compression Multipliers [Ph.D. thesis], Department of Electrical and Computer Engineering, University of Texas at Austin, Austin, Tex, USA, 2007.

[2] B. Parhami, Computer Arithmetic: Algorithms and Hardware Designs, Oxford University Press, New York, NY, USA, 2nd edition, 2010.

[3] C. S. Wallace, "A suggestion for a fast multiplier," IEEE Transactions on Electronic Computers, vol. EC-13, pp. 14-17, 1964.

[4] L. Dadda, "Some schemes for parallel multipliers," Alta Frequenza, vol. 34, pp. 349-356, 1965.

[5] V. G. Oklobdzija, D. Villeger, and S. S. Liu, "A method for speed optimized partial product reduction and generation of fast parallel multipliers using an algorithmic approach," IEEE Transactions on Computers, vol. 45, no. 3, pp. 294-306, 1996.

[6] K. C. Bickerstaff, E. E. Swartzlander, and M. J. Schulte, "Analysis of column compression multipliers," in Proceedings of the 15th IEEE Symposium on Computer Arithmetic, pp. 33-39, June 2001.

[7] W. J. Townsend, E. E. Swartzlander, and J. A. Abraham, "A comparison of Dadda and Wallace multiplier delays," in Advanced Signal Processing Algorithms, Architectures, and Implementations XIII, vol. 5205 of Proceedings of the SPIE, pp. 552-560, August 2003.

[8] H. Eriksson, P. Larsson-Edefors, M. Sheeran, M. Själander, D. Johansson, and M. Schölin, "Multiplier reduction tree with logarithmic logic depth and regular connectivity," in Proceedings of the IEEE International Symposium on Circuits and Systems, pp. 4-8, May 2006.

[9] M. Själander and P. Larsson-Edefors, "The case for HPM based Baugh-Wooley multipliers," Tech. Rep. 08-8, Chalmers University of Technology, Goteborg, Sweden, 2008.

[10] C. R. Baugh and B. A. Wooley, "A two's complement parallel array multiplication algorithm," IEEE Transactions on Computers, vol. 22, pp. 1045-1047, 1973.

[11] M. Själander and P. Larsson-Edefors, "High-speed and lowpower multipliers using the Baugh-Wooley algorithm and HPM reduction tree," in Proceedings of the 15th IEEE International Conference on Electronics, Circuits and Systems (ICECS '08), pp. 33-36, September 2008.

[12] V. G. Oklobdzija and D. Villeger, "Improving multiplier design by using improved column compression tree and optimized final adder in CMOS technology," IEEE Transactions on Very Large Scale Integration (VLSI) Systems, vol. 3, no. 2, pp. 292-301, 1995.

[13] P. F. Stelling and V. G. Oklobdzija, "Design strategies for optimal hybrid final adders in a parallel multiplier," Journal of VLSI Signal Processing Systems for Signal, Image, and Video Technology, vol. 14, no. 3, pp. 321-331, 1996.

[14] B. Ramkumar, H. M. Kittur, and P. M. Kannan, "ASIC implementation of modified faster carry save adder," European Journal of Scientific Research, vol. 42, no. 1, pp. 53-58, 2010.

[15] B. Ramkumar and H. M. Kittur, "Low-power and area-efficient carry select adder," IEEE Transactions on Very Large Scale Integration (VLSI) Systems, vol. 20, no. 2, pp. 371-375, 2012.

[16] B. Ramkumar and H. M. Kittur, "Optimal final carry propagate adder design for parallel multipliers," http://arxiv.org/ abs/1110.3584.

[17] Y. He, C. H. Chang, and J. Gu, "An area efficient 64-bit square root carry-select adder for low power applications," in 
Proceedings of the IEEE International Symposium on Circuits and Systems (ISCAS '05), vol. 4, pp. 4082-4085, May 2005.

[18] M. Själander and P. Larsson-Edefors, "High-speed and lowpower multipliers using the Baugh-Wooley algorithm and HPM reduction tree," in Proceedings of the 15th IEEE International Conference on Electronics, Circuits and Systems (ICECS '08), pp. 33-36, September 2008.

[19] S. R. Kuang, J. P. Wang, and C. Y. Guo, "Modified booth multipliers with a regular partial product array," IEEE Transactions on Circuits and Systems II, vol. 56, no. 5, pp. 404-408, 2009. 

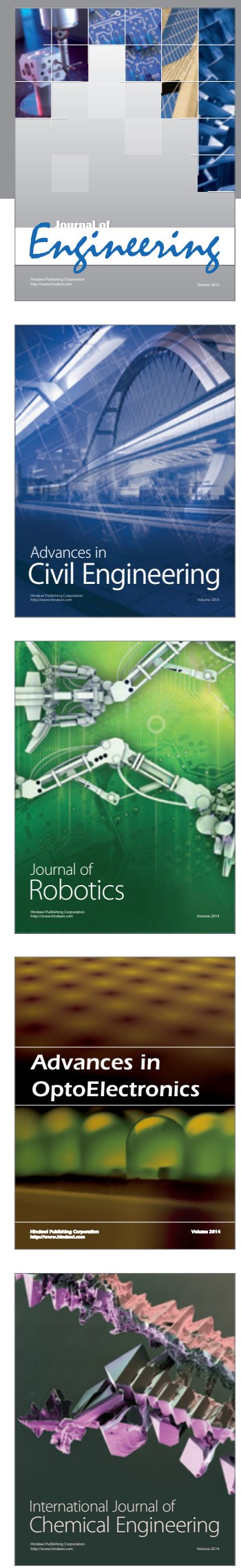

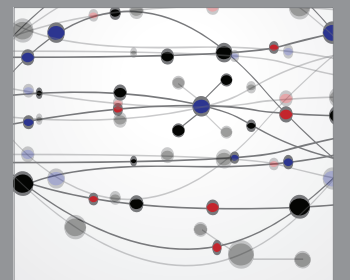

The Scientific World Journal
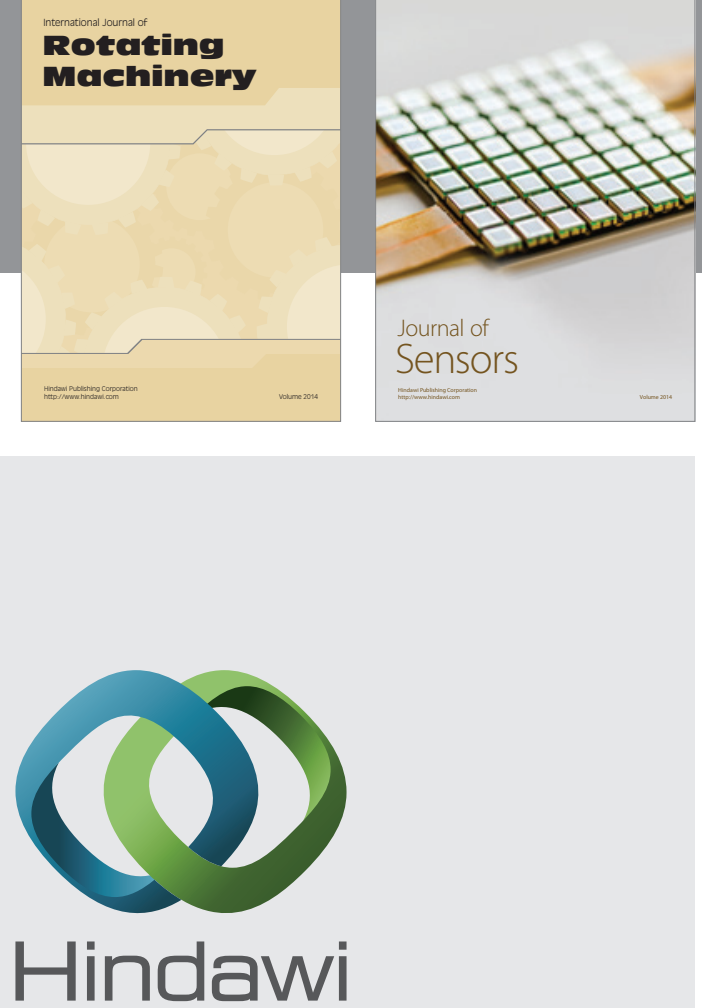

Submit your manuscripts at http://www.hindawi.com
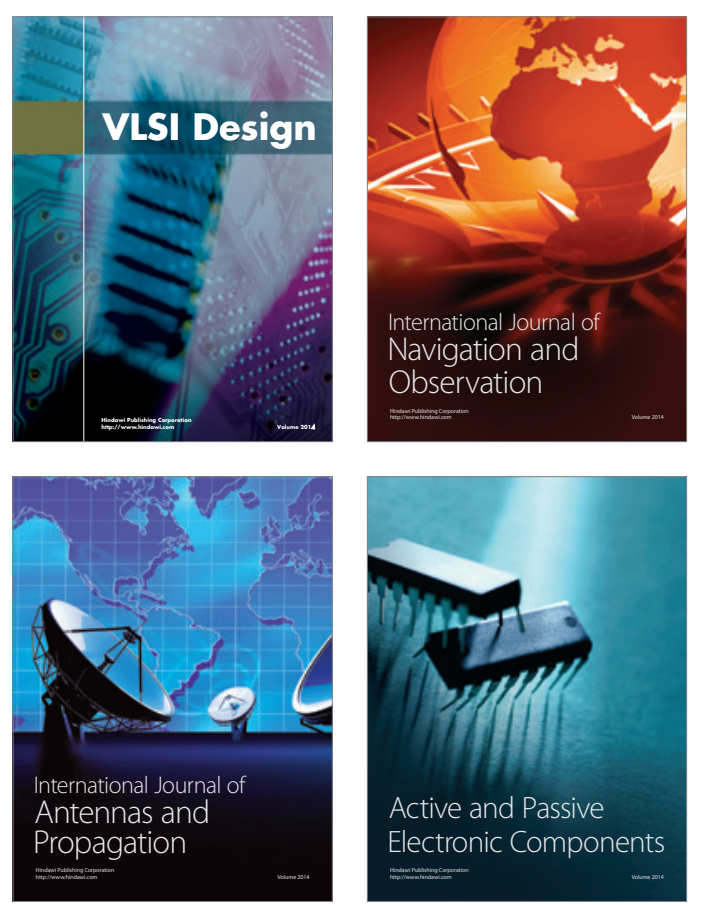
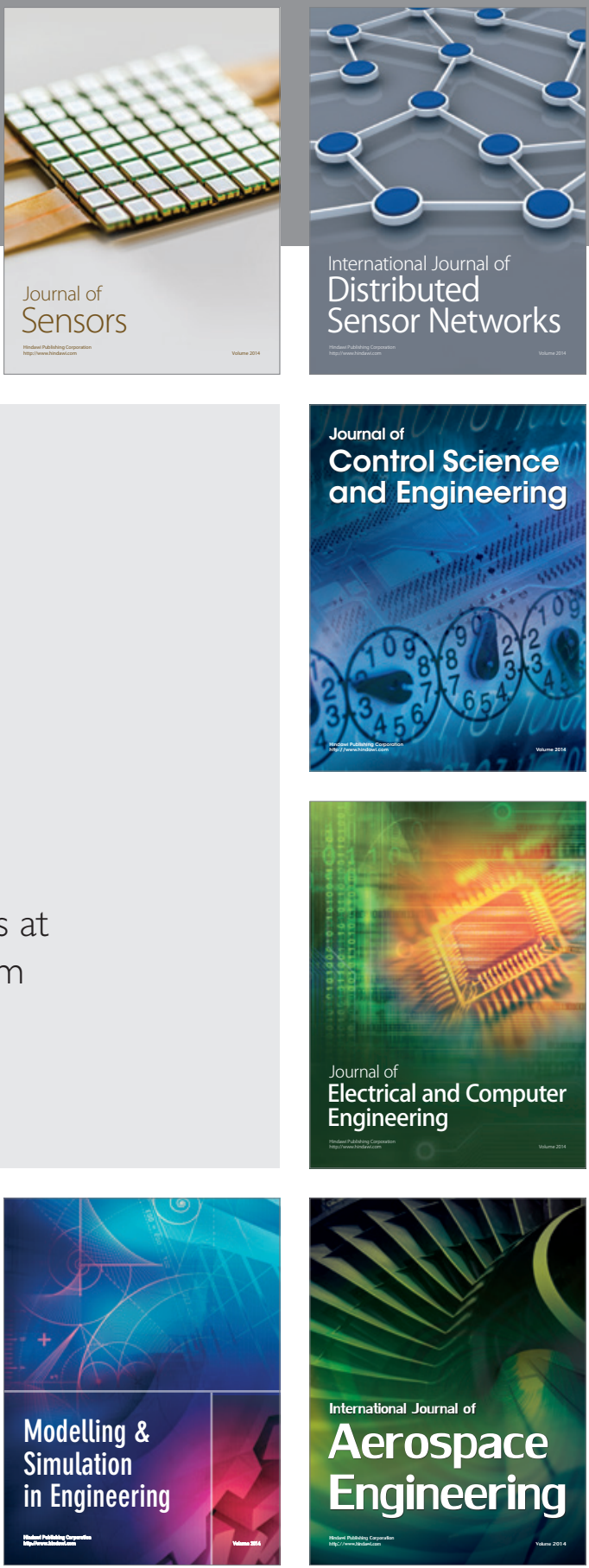

Journal of

Control Science

and Engineering
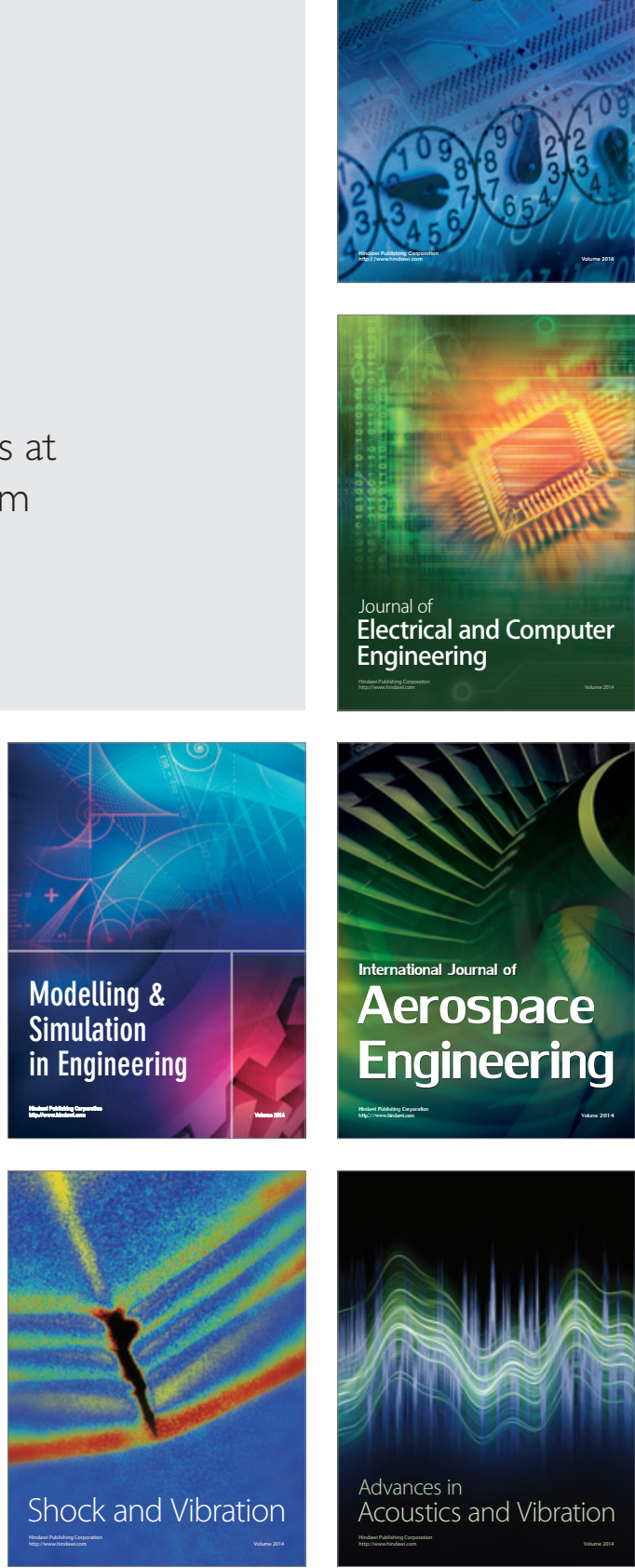